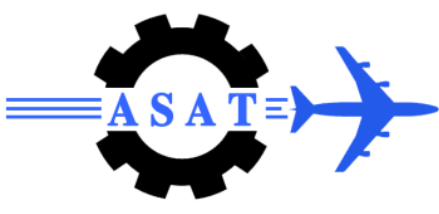

\title{
Peak-to-Average Power Ratio Reduction for Single Carrier- Frequency Division Multiple Access using Repeated Clipping and Filtering
}

\author{
H.E.A. Hassan ${ }^{*}$, F.A.K. Al-fuhaidy ${ }^{\dagger}$, Kh. El-barbary ${ }^{\ddagger}$
}

\begin{abstract}
A single carrier frequency division multiple access (SC-FDMA), is a technique that has a similar performance and essentially the same overall complexity as those of orthogonal frequency division multiple access (OFDMA). The main advantage of SC-FDMA over OFDMA is its lower peak-to-average power ratio (PAPR). In this paper, an enhancement of a SC-FDMA by decreasing the PAPR is addressed. An efficient repeated clipping and filtering (RCF) algorithm is applied to a SC-FDMA system, which results in a significant PAPR reduction. System performance remains the same after applying the RCF algorithm. Simulations show the proposed scheme provides a bit error as that of the conventional system. That is, due to the selection of the optimum clipping ratio (CR). For more accurate validation, simulations of the conventional system and the proposed system have been performed using different types of channels. The proposed scheme provides a PAPR reduction by a gain of approximately $4 \mathrm{~dB}$ with a slight increase of complexity.
\end{abstract}

\section{Introduction}

Recently, wireless communication systems offer a high data rate. Orthogonal frequency division multiplexing (OFDM), which is a multicarrier communication technique, has received a lot of attention in the last few years. This is due to its ability to overcome the frequency-selective fading which is common in wireless broadband communications [1]. OFDMA is an extension of OFDM to accommodate multiple simultaneous users. Despite the benefits of OFDM and OFDMA, they suffer a number of drawbacks such as the high PAPR [2]. The high PAPR requires system components with a large linear range capable of accommodating the signal. Otherwise, the nonlinear distortion occurs, which results in a loss of subcarrier orthogonality and degrades the system performance. Lower PAPR greatly benefits the mobile terminal in terms of transmitted power efficiency. SC-FDMA is adopted as a possible air interface, especially in uplink broadband communications. The main advantage of SC-FDMA compared to multi-carrier systems is its low PAPR, in addition to the advantages of OFDMA [3]. However, a SC-FDMA system performance can be enhanced by decreasing the PAPR. Many PAPR reduction techniques for multi- carrier systems are surveyed in [4], which may be used by single-carrier systems. PAPR reduction techniques can be classified into two categories. The first one is the signal distortion techniques which introduces distortion to signals and causes degradation in the performance such as clipping algorithm. The second category is called symbol scrambling, which may not affect the system

\footnotetext{
*Egyptian Armed Forces, Egypt, hossameldin_aboubakr@hotmail.com

† Yemen Armed Forces, farouqakh@gmail.com

Modern Academy, Staff member, khbar2000@yahoo.com.
} 
performance but has an overhead and significantly increased complexity such as Partial Transmit Sequence (PTS), SeLective Mapping (SLM) and other techniques presented in [56]. Clipping is the simplest effective algorithm that can be used for PAPR reduction. But, it introduces additional clipping noise which degrades the system performance. Clipping introduces in-band distortion and out-of-band radiation. It is worth noting that out of band radiation can be solved by filtering the clipped signal [7-8]. Clipping and filtering may cause some peak regrowth letting the signal samples to exceed the clipping level at some points. This can be solved by repeated clipping and filtering [8]. In this paper, an optimum clipping ratio is selected to have no effect on the system performance in terms of BER.

The remainder of this paper is organized as follows; Section 2 provides an overview of SCFDMA system. Section 3, describes the PAPR problem in broadband communications. An analysis of the suggested system is presented in Section 4. Simulation results and discussions are presented in Section 5. Finally, the conclusions and the recommendations for future work are presented in Section 6.

\section{SC-FDMA System Model}

The third generation partnership project (3GPP), investigates a modified form of OFDMA for uplink transmissions by the "long-term evolution (LTE)" of cellular systems [9-10]. The block diagram describing the SC-FDMA system is shown in Figure 1 [11].

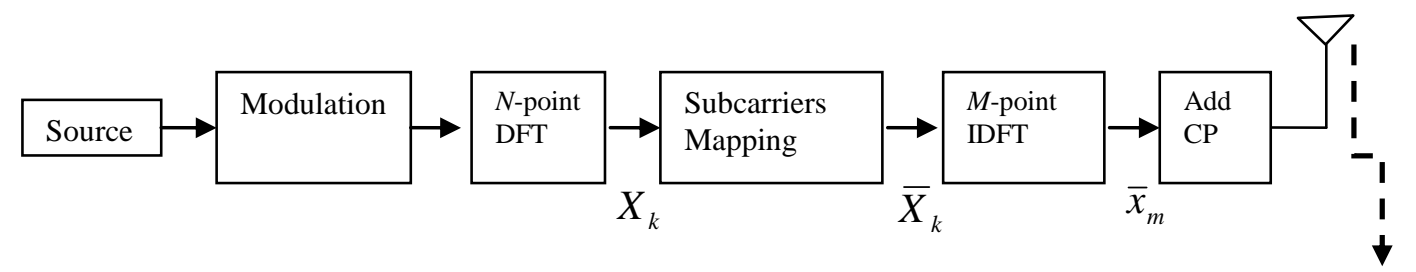

(a) Transmitter of the user $u$

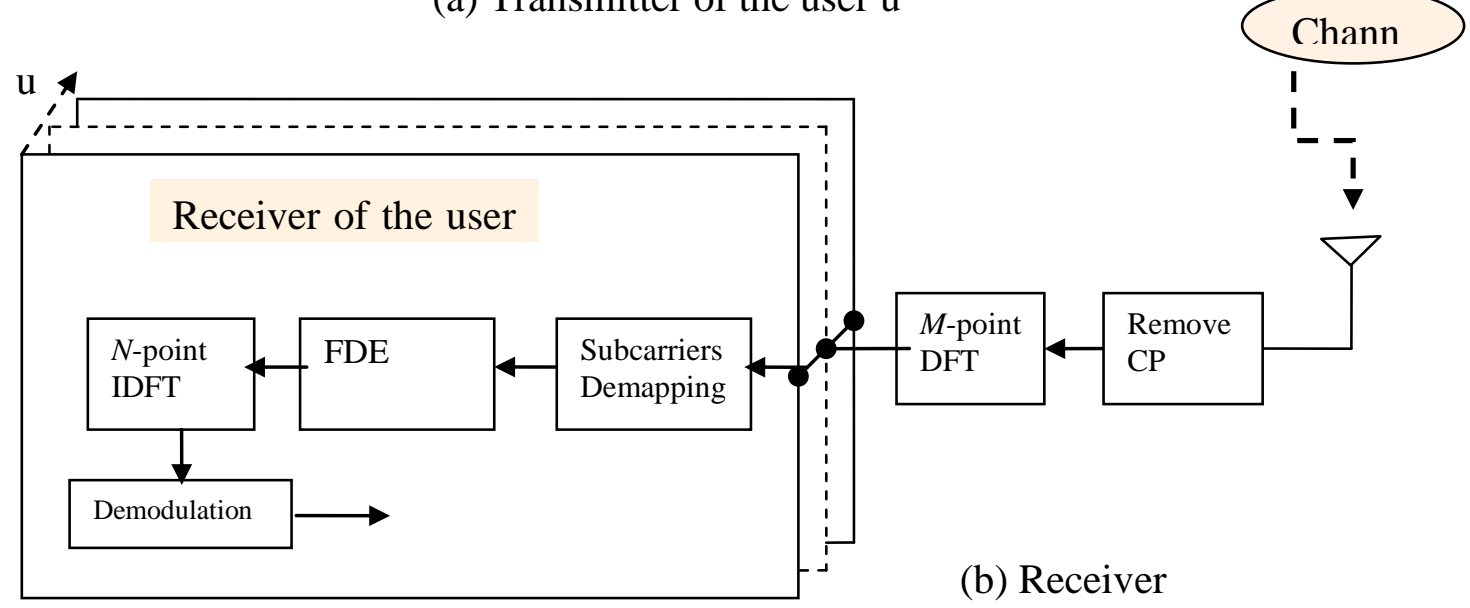

\section{Figure 1: Structure of DFT SC-FDMA system over a frequency selective channel.}

At the transmitter, binary input data passes through one of many possible modulation techniques such as QAM, or M-QAM, introducing symbols. A discrete Fourier transform (DFT) operation is performed on each block of $N$ symbols. Then, subcarriers are mapped in the frequency domain. There are three types of mapping, localized, interleaving, or distributed mapping. The inverse DFT is performed on each block of $M$ subcarriers. After that, a cyclic 
prefix (CP) of $N c$ symbols is added to the resulting signal. The length of the $\mathrm{CP}$ must be greater than the maximum delay spread of the channel to combat the inter-block interference (IBI) [3-11]. The signal after DFT can be expressed as follows:

$$
X_{k}=\sum_{n=0}^{N-1} x_{n} e^{-\frac{j 2 \pi}{N} n k}
$$

where $x_{n}$ is an $N \times 1$ vector containing the modulated data symbols. $N$ is a DFT length. After the IDFT, the signal can be expressed as follows:

$$
\bar{x}_{m}=\frac{1}{M} \sum_{l=0}^{M-1} \bar{X}_{l} e^{\frac{j 2 \pi}{M} m l}
$$

where $\bar{X}_{l}$ represents the frequency domain samples after subcarrier mapping, $M$ is the IDFT length (number of subcarriers $M>N$ ) and $\bar{x}_{m}$ represents the time symbols after the IDFT. The baseband channel impulse response can, then, be expressed as follows [12-13]:

$$
h(t)=\sum_{l=0}^{L-1} h_{l} \delta\left(t-\tau_{l}\right)
$$

where $h_{l}$ and $\tau_{l}$ are the complex fading and the propogation delay of the $l^{\text {th }}$ path respectively, and $L$ is the number of the multipath components of the channel impulse response $h(t)$. At the receiver, the CP is removed from the received signal. An M-point DFT is performed on the received signal, to get the frequency domain signal. Then, Frequency Domain Equalization (FDE) and subcarrier demapping are performed. An N-point IDFT is performed on the resulting signal. Finally, demodulation is performed. After removing CP, the received signal can be expressed as follows:

$$
r=H \bar{x}+n
$$

where $\bar{x}$ is an $M \times 1$ vector representing the block of the transmitted symbols and $r$ is an $M \times 1$ vector representing the received symbols. $n$ is an $M \times 1$ vector describing the additive noise. The matrix $H$ is an $M \times M$ circulant matrix describing the multipath channel and can be expressed as follows [13]:

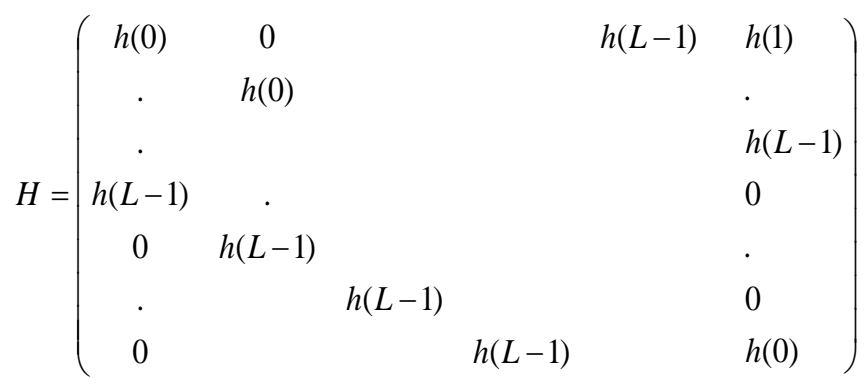

The circulant $H$ matrix can be efficiently diagonalised by the fast fourier transform $(\Psi)$ and inverse fast fourier transform $\left(\Psi^{-1}\right)$. It can be written as follows [13]:

$H=\Psi^{-1} \Lambda \Psi$ 
where $\Lambda$ is an $M \times M$ diagonal matrix containing the fast fourier transform of the circulant sequence of $H$. The FDE complex coefficients $\omega(m)$ can be derived according to the minimum mean square (MMSE) criterion as follows [14]:

$$
\omega(m)=\frac{\Lambda^{*}(m, m)}{|\Lambda(m, m)|^{2}+1 / S N R}
$$

where $S N R$ is the signal-to-noise ratio. The advantage of the frequency domain equalization is its low computational complexity and its disadvantage is that require insertion of $\mathrm{CP}$ which reduces the data rate.

\section{PAPR Problem Definition}

The PAPR in SC-FDMA is required to be as small as possible. The output of the IDFT block leads to the Gaussian distribution of different peak amplitude values. This causes some challenges to the amplifier design as, in a cellular system; one should aim for maximum power amplifier efficiency to achieve minimum power consumption. Figure 2 illustrates how a signal with a higher envelope variation requires the amplifier to use additional input backoff power compared to a small envelope variation. The amplifier must stay in the linear area with the use of extra back-off power in order to prevent problems to the output signal and spectrum mask. The use of additional back-off leads to a reduced amplifier power efficiency or a smaller output power. This either causes the uplink range to be shorter, when the same average output power level is maintained or, the battery energy is consumed faster due to higher amplifier power consumption. The latter is not considered a problem in fixed applications where the device has a large volume and is connected to the main supply, but for small mobile devices running on their own batteries it creates more challenges.
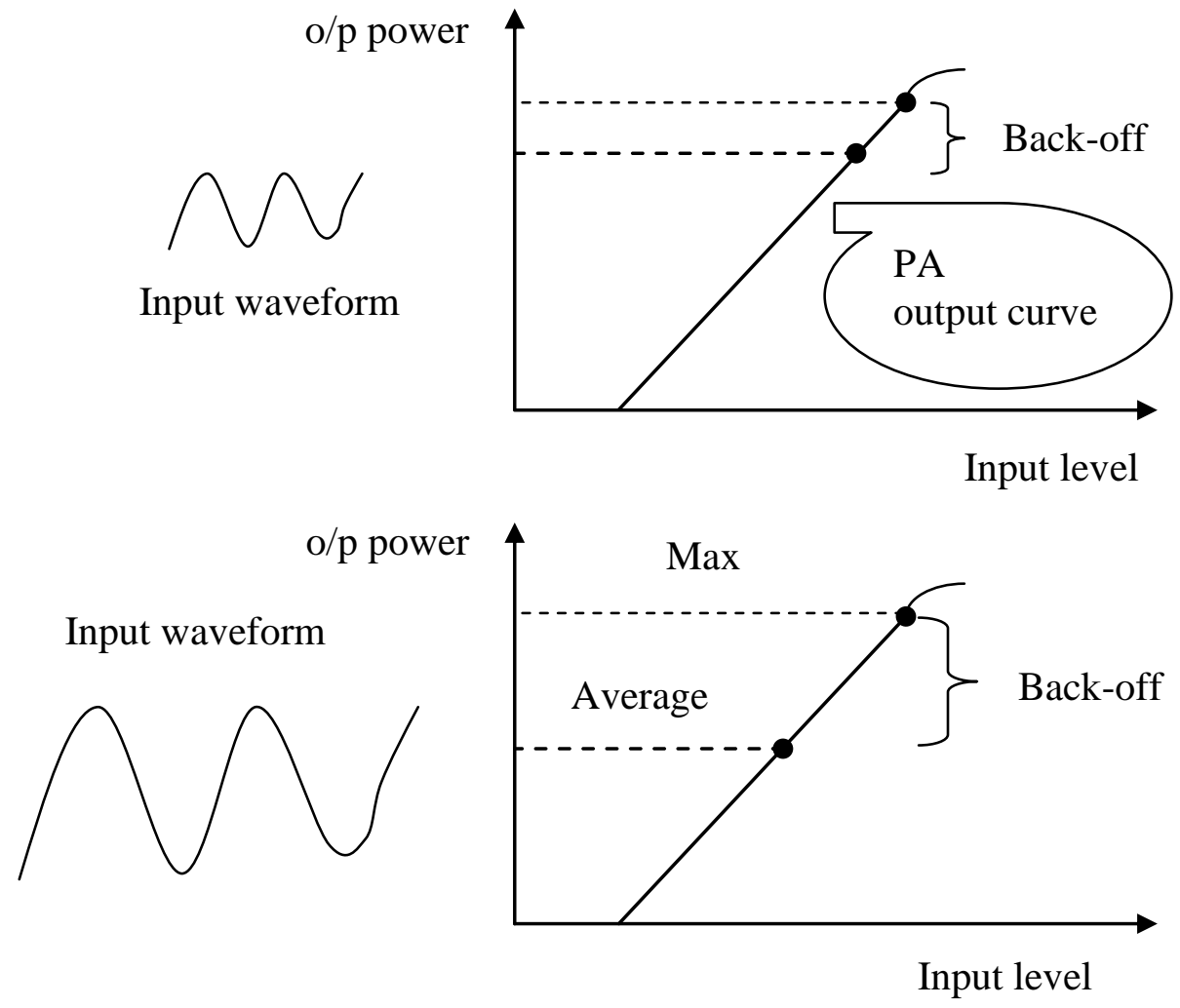

Figure 2: Power amplifier back-off requirement for different input waveforms. 
The high PAPR causes the peaks to enter the saturation region of the power amplifier (PA) which results in a significant in-band distortion and out-of-band radiation when a signal passes through a nonlinear device such a transmitter device (PA). The in-band distortion degrades the performance by increasing BER. Moreover, the out-band radiation results in an unacceptable adjacent channel interference [15-16]. The PAPR is defined as the ratio of peak power to average power of the transmitted signal in a given transmission block, and is given by:

$$
P A P R=\frac{P_{p e a k}}{P_{a v}}=\frac{\left.\left.\max _{0 \leq m \leq M-1}\left(\mid \bar{x}_{m}\right)\right|^{2}\right)}{1 / M \sum_{m=0}^{M-1}\left|\bar{x}_{m}\right|^{2}}
$$

The cumulative distribution function (CDF) of the PAPR is one of the most performance measures for different PAPR reduction techniques. Most researches use complementary CDF (CCDF) instead of CDF [17-18]. The CCDF of the PAPR denotes the probability that the PAPR of a data block exceeds a given threshold PAPR $>$ PAPR th. $_{\text {. }}$.

\section{The Proposed LFDMA System with Clipping and Frequency Filtering}

This section presents the proposed SC-FDMA system with RCF technique. Figure 3 shows the block diagram of the proposed SC-FDMA. This structure combines the repeated clipping and frequency filtering (RCF).

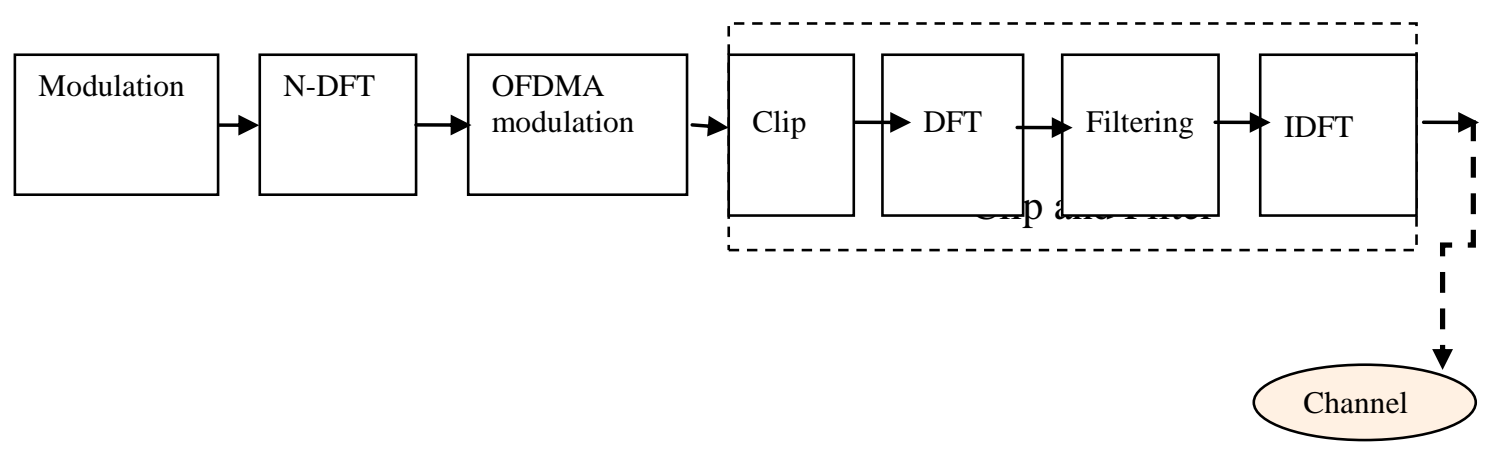

\section{Figure 3: Structure of DFT SC-FDMA transmitter system combined RCF algorithm.}

The simplest approach to reduce the PAPR of SC-FDMA is to clip the high amplitude peaks. Such clipping introduces in-band distortion and out-of-band radiation. Out-of-band radiation can be reduced by filtering. Filtering results in some peak regrowth as in other clip and filter technique. Repeated clipping-and-filtering operations can be used to reduce the overall peak regrowth [8]. In this paper, the in-band clip distortion is improved by selecting an optimum clipping ratio. That results in no degradation in system performance. The clipping function is performed in the time domain, and the process is described by the following expression:

$$
x^{c}{ }_{k}= \begin{cases}x_{k} & \left|x_{k}\right|<=\mathrm{A} \\ A e^{\phi\left(x_{k}\right)} & \left|x_{k}\right|>\mathrm{A}\end{cases}
$$


where $x^{c}{ }_{k}$ is the clipped signal, $x_{k}$ is the base-band signal, $A$ is the peak amplitude and $\phi\left(x_{k}\right)$ is the phase of the baseband signal which has no change due to clipping. The clip ratio $(\mathrm{CR})$ is calculated using this formula:

$C R=\frac{A^{2}}{P_{a v}}$

where $P_{a v}$ is the average of the signal (expectation of the energy signal). It is expected that the proposed system provides a good enhancement in terms of PAPR with some additional complexity, by introducing DFT, filtering and IDFT components. The method of applying $\mathrm{RCF}$ algorithm is summarized as follows:

1. The binary input signal is passed through the modulator, introducing blocks of data symbols.

2. Each block of data symbols are arranged in parallel and then converted to frequency domain by DFT operation.

3. After DFT spread, OFDMA modulation is applied to the block of symbols which includes subcarrier mapping and IDFT operation.

4. After that, the RCF technique is applied, which starts by clipping the amplitude of the signal in the time domain based on the optimum selected CR. This optimum CR is selected by simulation of BER with CR for different signal-to-noise ratio (SNR). The optimum CR is selected such that the BER is not degraded. Then, the block is transformed to the frequency domain and a low-pass filtering is applied. Finally, filtered data is converted back to time domain using IDFT, and the process is repeated until we get the required clipping threshold.

\section{Simulations and Results}

Computer simulations are performed using MATLAB to validate the results provided in the next subsections. Localized mapping which is used by LTE in $3^{\text {rd }}$ generation partnership project (3GPP) for uplink transmission [19] is implemented. The simulation parameters are listed in Table 1.

Table.1. Simulation Parameters

\begin{tabular}{l|l|l}
\hline & Description & Parameters \\
\hline \hline \multirow{4}{*}{ Transmitter } & System bandwidth & $5 \mathrm{MHz}$ \\
\cline { 2 - 3 } & Modulation & QPSK \\
\cline { 2 - 3 } & $\mathrm{CP}$ & 20 samples \\
\cline { 2 - 3 } & Transmitter IDFT size & $\mathrm{M}=512$ \\
\cline { 2 - 3 } & Subcarrier spacing & $9.765625 \mathrm{kHz}$ \\
\cline { 2 - 3 } & SC-FDMA input block size & 128 symbols \\
\cline { 2 - 3 } & Subcarrier mapping & Localized \\
\hline \multirow{4}{*}{ Channel } & Channel model & $\begin{array}{l}\text { Vehicular A outdoor channel and Fixed } \\
\text { channel }\end{array}$ \\
\cline { 2 - 3 } & Noise environment & AWGN \\
\hline \multirow{2}{*}{ Receiver } & Channel estimation & Perfect, and MMSE channel estimator \\
\cline { 2 - 3 } & Equalization & MMSE \\
\hline \hline
\end{tabular}


Performance evaluations of the proposed SC-FDMA are provided under different wireless channels. The SUI3 channel, the vehicular A outdoor channel, and the additive white Gaussian Noise (AWGN) channel are used in the simulations. The BER is evaluated by the Monte Carlo simulation method with $10^{4}$ run times. The conventional SC-FDMA scheme is also simulated for comparison purpose. One of six channel models adopted by IEEE 802.16a standard for evaluating the performance of broadband wireless systems in the $2-11 \mathrm{GHz}$ band is the SUI3 channel [20]. It has three Rayleigh fading taps at delays of $0,0.5$ and $1 \mathrm{~ms}$, with relative powers of 0,25 , and $210 \mathrm{~dB}$, respectively. The vehicular A outdoor channel has six Rayleigh fading taps at delays of 0,310, 710, 1090, 1730 and $2510 \mathrm{~ns}$, with relative powers of $0,21,29,210,215$ and $220 \mathrm{~dB}$, respectively [21]. The vehicular A outdoor channel has a mobile speed of $120 \mathrm{~km} / \mathrm{h}$. A mobile speed of $120 \mathrm{~km} / \mathrm{h}$ corresponds to a Doppler spread of $223 \mathrm{~Hz}$ for a carrier frequency of $2 \mathrm{GHz}$. The BER is evaluated by the Monte Carlo simulation method with $10^{4}$ runs. The conventional SC-FDMA scheme is also simulated for comparison purpose.

\section{Simulations for Optimum CR}

In order to select the optimum CR, many scenarios of simulation are performed for different parameters. The BER versus CR for different SNR, QPSK modulation under vehicular channel is simulated and shown in Figure 4

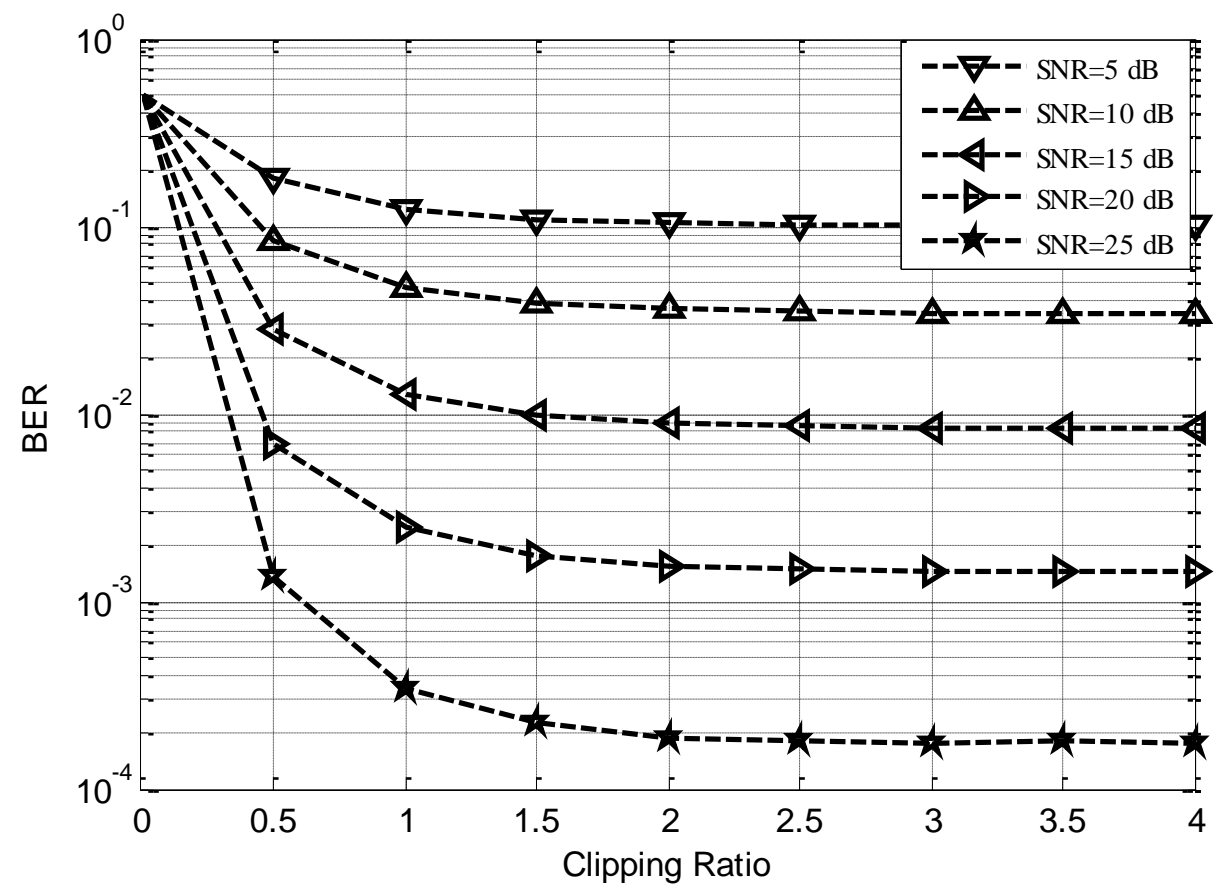

Figure 4: Bit error versus clipping ratio of SC-FDMA for different SNR values, QPSK modulation, and Vehicular channel.

It is clear that for values of CR greater than or equal to $2 \mathrm{~dB}$, the system performance, BER, approaches to be constant. Since, smaller CR results in smaller PAPR, we choose CR equal to $2 \mathrm{~dB}$ as the optimum. Moreover, for more verification Figure 5 shows the simulation of the system with fixed channel. 


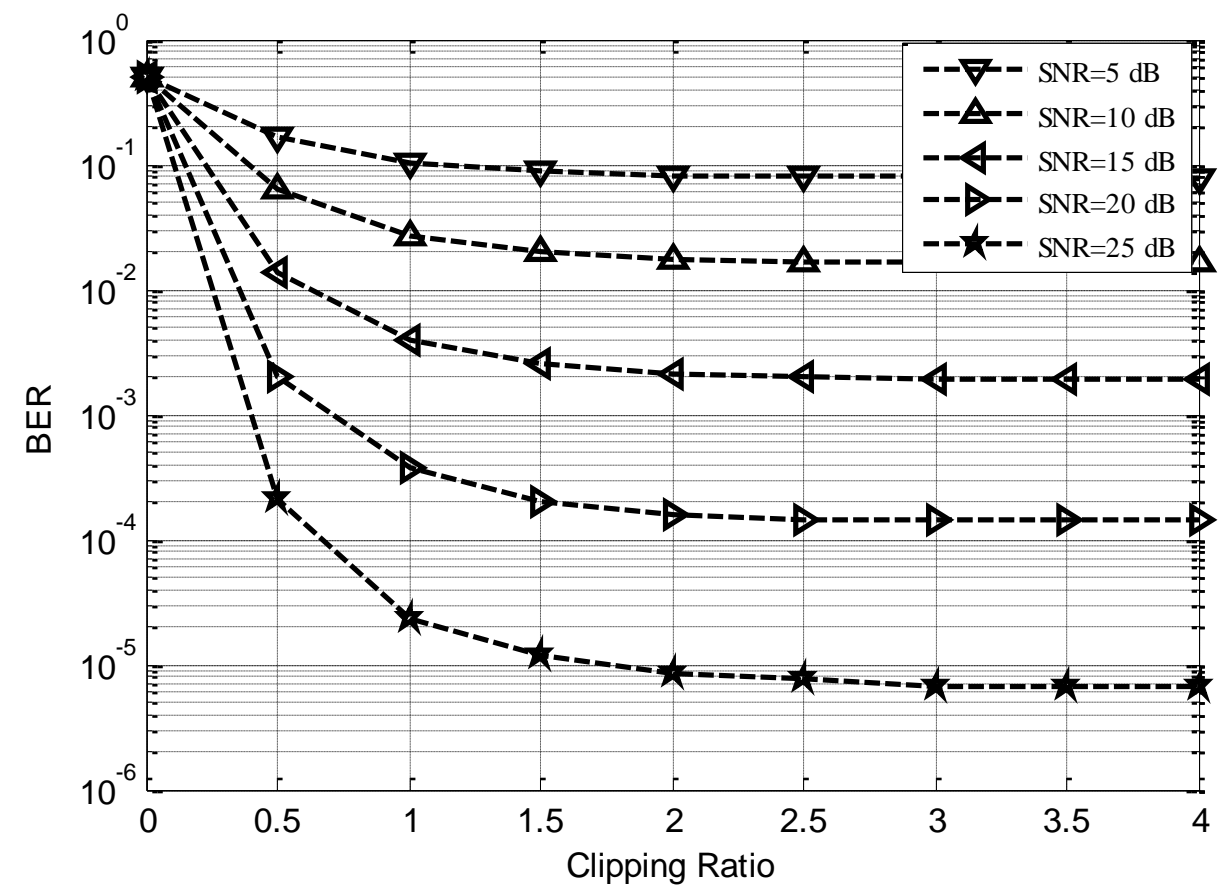

Figure 5: Bit error vs. clipping ratio of SC-FDMA for different signal-to-noise ratio, QPSK modulation, and fixed channel.

As shown in the previous figure, the behavior of the proposed scheme with fixed channel is the same as that of the vehicular channel. This indicates that the selected CR as optimum is not affected with changing the channel.

\section{Simulations of BER versus SNR at the Optimum CR}

In this subsection, simulation of BER versus $S N R$ at the optimum $C R=2 \mathrm{~dB}$ is presented. To verify that the selected $C R$ is the optimum, simulations of the BER versus SNR for the proposed scheme with clipping is compared to the conventional scheme is shown in Figure 6.

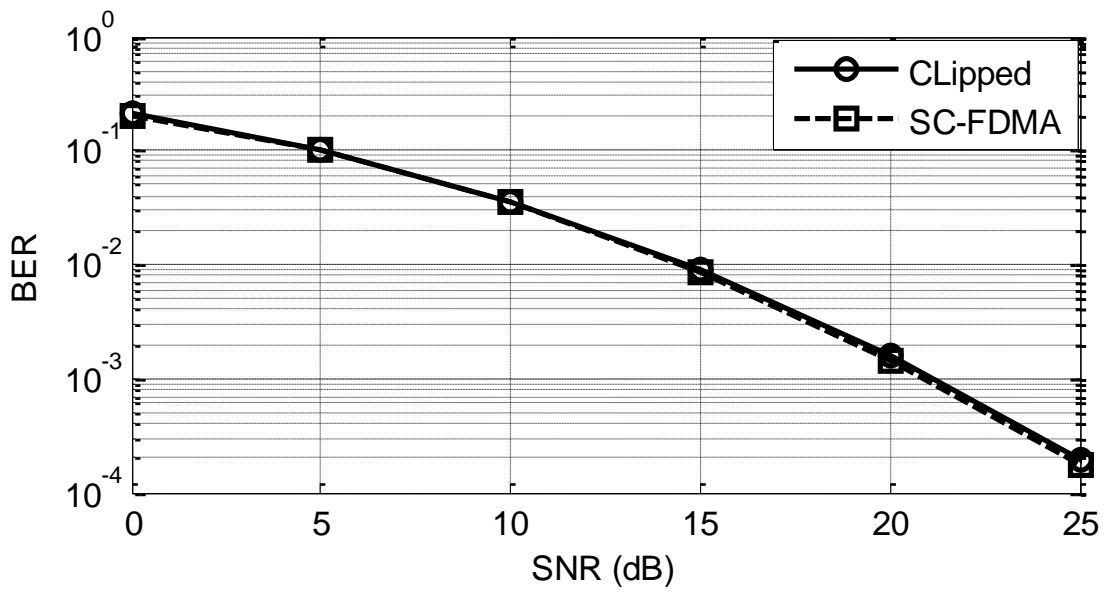

Figure 6: Bit error versus SNR of SC-FDMA for $\mathrm{CR}_{\mathrm{opt}}=2 \mathrm{~dB}$, QPSK modulation and Vehicular channel.

The previous figure compares the proposed scheme to the conventional scheme under vehicular channel. It is shown that BER for the proposed system matches the conventional system. That is due to choosing the optimum CR that does not affect the performance. Moreover, the proposed scheme is compared to the conventional scheme under fixed channel as shown in figure 7. 


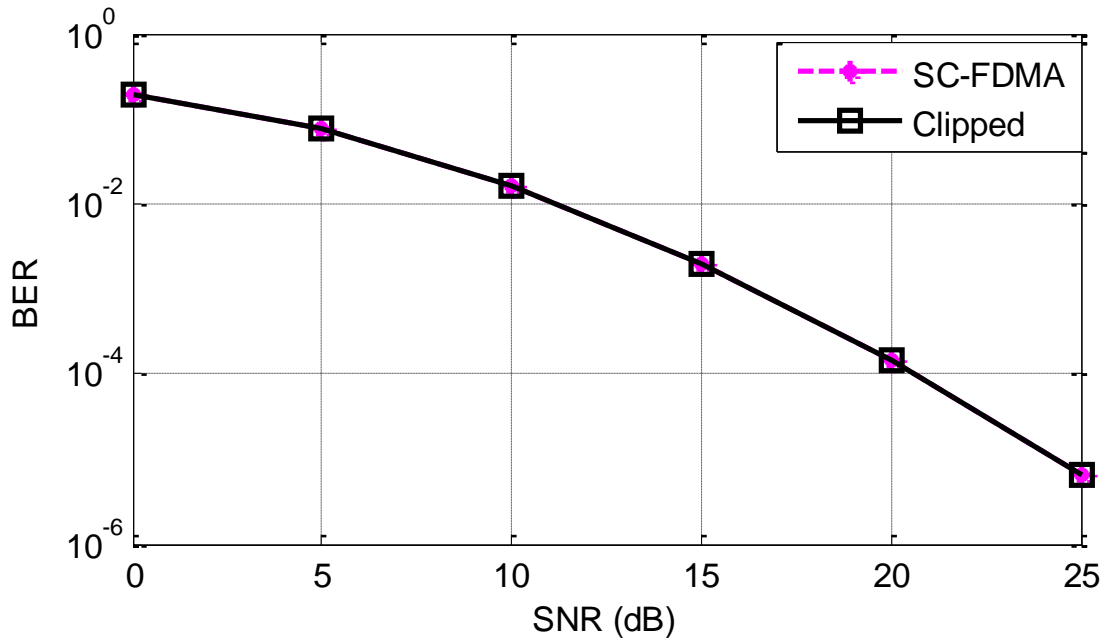

Figure 7: Bit error vs. signal-to-noise ratio of SC-FDMA for CR=2, QPSK modulation, and fixed channel.

It is shown that BER for the proposed system the same as the conventional system. And there is no effect due to the clipping of the signal. As shown in the previous figures, the selected clipping ratio does not affect the system performance. However, it will provide an enhancement in PAPR as it will be shown in next subsection.

\section{Peak-to-Average Power Ratio Reduction}

The proposed SC-FDMA system combined RCF algorithm is compared to the conventional SC-FDMA system in terms of PAPR. PAPR represents the fluctuation of the signal which is the peak to average signal power. To evaluate the PAPR of individual system configurations, a transmission of $10^{4}$ blocks of symbols is simulated. After calculating PAPR for each block, the data is presented as an empirical CCDF (Complementary Cumulative Distribution Function). The CCDF is the probability that PAPR is higher than a certain PAPR value, $\mathrm{PAPR}_{\mathrm{th}}\left(\mathrm{Pr}\left\{\mathrm{PAPR}>\mathrm{PAPR}_{\mathrm{th}}\right\}\right)$. Figure 8 simulates the CCDF for the PAPR of the conventional SC-FDMA and the proposed system with QPSK modulation.

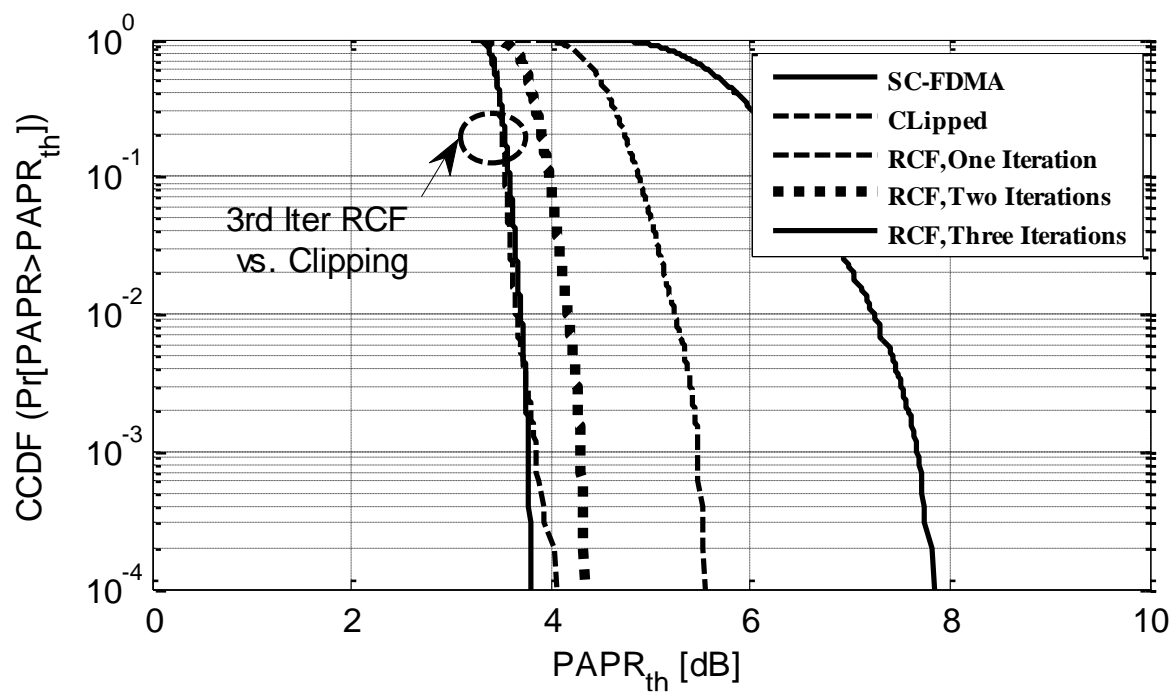

Figure 8: CCDF of PAPR for SC-FDMA for 1, 2, 3 iterations of RCF, QPSK modulation. 
It is shown in Figure 6 that the proposed system outperforms the conventional system in terms of PAPR. For example, at 0.001 CCDF, the PAPR for the proposed system provides a significant and comparable enhancement to PAPR of the conventional system, by an enhancement gain of approximately $4 \mathrm{~dB}$. However, the system has no performance degradation as shown in the previous subsection. That is due to the selection of the optimum CR.

Finally, Figure 9 shows the CCDF for PAPR of the proposed system compared to SC-FDMA system. Different CR values 2, 2.5 and $3 \mathrm{~dB}$ are used.

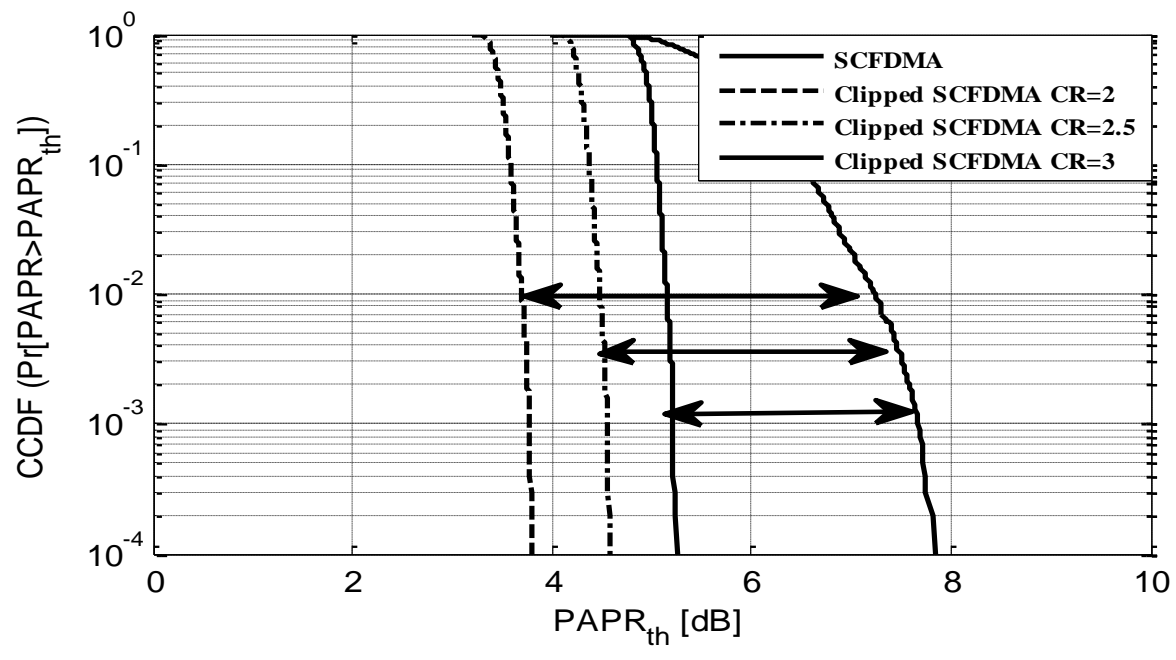

Figure 9: CCDF of PAPR for LFDMA and CR=2, 2.5 and $3 \mathrm{~dB}$, QPSK modulation.

There is a significant PAPR reduction for different $\mathrm{CR}$ values. As the clipping ratio increases the PAPR reduction decreases. This provides a freedom for selecting the CR that provides the best BER and therefore, reduces the PAPR of the signal.

\section{Conclusions and Future Work}

It has been shown that the SC-FDMA is a promising alternative uplink transmission system. That is due to its lower PAPR compared to OFDMA system. In this work, a significant PAPR reduction on SC-FDMA is provided. In this paper, a RCF algorithm is applied for PAPR, which uses clipping and frequency domain filtering for PAPR reduction combined with SCFDMA system. Simulation results show that the proposed scheme outperforms the conventional system, in terms of PAPR. The optimum CR which does not degrade the system performance is selected and verified by simulations. The RCF algorithm provides a good and significant PAPR reduction up to $4 \mathrm{~dB}$. However, RCF technique increases the system computation complexity by adding DFT, IDFT and filtering operations. It is recommended as a future work, the use of a one iteration clipping and filtering rather than repeated clipping and filtering as in [22]. 


\section{References}

[1] L J Cimini, Jr, "Analysis and Simulation of a Digital Mobile Channel Using Orthogonal Frequency Division Multiplexing," IEEE Trans. Communication., vol. 33, No. 7, July 1985, pp. 665-675.

[2] D FALCONER, S L ARIYAVISITAKUL, A BENYAMIN-SEEYAR, B EIDSON B "Frequency domain equalization for single-carrier broadband wireless systems," IEEE Commun. Mag., 2002, 40, pp. 58-66.

[3] H. G. Myung, J. Lim and D. J. Goodman, "Single Carrier FDMA for Uplink Wireless transmission," Proceeding of the IEEE Vehicular Technology Magazine. , vol. 1, No.3, 2006.

[4] S. H. Han and J. H. Lee, "An Overview of Peak-to-Average Power Ratio Reduction Techniques for Multicarrier Transmission," IEEE Wireless Communications, vol. 12, No. 2, 2005, pp. 56-65.

[5] ZID Souad and B. Ridha, "Low-Complexity PAPR Reduction Schemes Using SLM and PTS Approaches for Interleaved OFDMA," IEEE, 2009.

[6] Seng-Hung Wang, Jia-Cheng Xie and Shih-Peng Li, "A Low-Complexity SLM PAPR Reduction Scheme for Interleaved OFDMA Uplink," IEEE GLOBECOM, 2009.

[7] Yue Xiao, Yongrui Peng and Shaoqian Li "PAPR Reduction for Interleaved OFDMA with Low Complexity," ICICS, 2007.

[8] J. Armstrong, "Peak-to-Average Power Reduction for OFDM by Repeated Clipping and Frequency Domain Filtering," Electron. Lett., vol. 38, Feb. 2002, pp. 246-247.

[9] H. Ekström, A. Furuskär, J. Karlsson, M. Meyer, S. Parkvall, J. Torsner, and M. Wahlqvist, "Technical Solutions for the 3G Long-Term Evolution," IEEE Commun. Mag., vol. 44, no. 3, pp. 38-45, Mar. 2006.

[10] 3rd Generation Partnership Project (3GPP); Technical Specification Group Radio Access Network; Physical Layer Aspects for Evolved UTRA, http://www.3gpp.org.[11] F.S. Al-kamali, M.I. Dessouky, B.M. Sallam, F. Shawki and F.E. Abd El-Samie, "Tranceiver Scheme for Single-Carrier Frequency Division Multiple Access Implementing the Wavelet Transform and Peak-to-Average power Ratio reduction methods," IET Comm, 2010, Vol 4, Iss. 1, pp. 69-79.

[12] F ADACHI, D GARGE, S TAKAOKA and K TAKEDA "Broadband CDMA techniques," IEEE Wirel. Comm, 2005, 12, (2), pp. 8-18.

[13] F S AL-KAMALI, M I DESSOUKY, B M SALLAM and F E EL-SAMIE "Performance evaluation of cyclic prefix CDMA systems with frequency domain interference cancellation,” Digital Signal Process. J., 2009, 19, (1), pp. 2-13

[14] Y C LIANG "Block-iterative GDFE (BI-GDFE) for CPCDMA and MC-CDMA," Proc. IEEE VTC, 2005, 5, (6), pp. 3033-3037.

[15] A. S. Baiha, M. Singh, A. J. Goldsmith, and B. R. Saltzberg, "A new approach for evaluating clipping distortion in multicarrier systems," IEEE J. Select Areas Commun., vol. 20, No. 5, pp. 1037-1046, June 2002.

[16] Xiaodong Li and Leonard J "Effects of Clipping and Filtering on the Performance of OFDM," IEEE Comm Letters, VOL. 2, NO. 5, MAY 1998.

[17] H. G. Myung, J. Lim and D. J. Goodman, "Peak-to-Average power Ratio of Single Carrier FDMA Signals with Pulse Shaping," Proceeding of the IEEE PIMRC, pp. 1- 5, Sep. 2006.

[18] G. Huang, A. Nix and S. Armour, "Impact of radio resource allocation and pulse shaping on PAPR of SC-FDMA signals," proceeding of the IEEE 18th International Symposium on Personal, Indoor and Mobile Radio Communications (PIMRC'07), Athens, pp. 1-5, 3-7 Sep. 2007. 
[19] H. G. Myung and D. J. Goodman, "Single Carrier FDMA A new Air Interface for Long Term Evaluation," Wiley Series on Wireless Communications and Mobile Computing, 2008.

[20] V ERCEG, K V S HARI, M S SMITH and ET AL "Channel models for fixed wireless applications," IEEE 802.16a cont. IEEE 802.16.3c-01/29r1, February 2001.

[21] Alpha Concept Group: "Wideband direct sequence CDMA (WCDMA) evaluation document (3.0)," Tdoc SMG 905/97, Madrid, Spain, December 1997.

[22] A K Gurung, F S Al-Qahtani, A Z Sadiq and Z M Hussain " One-Iteration-Clipping Filtering (OICF) scheme for PAPR reduction of OFDM signal,” ICATC,2008. 